
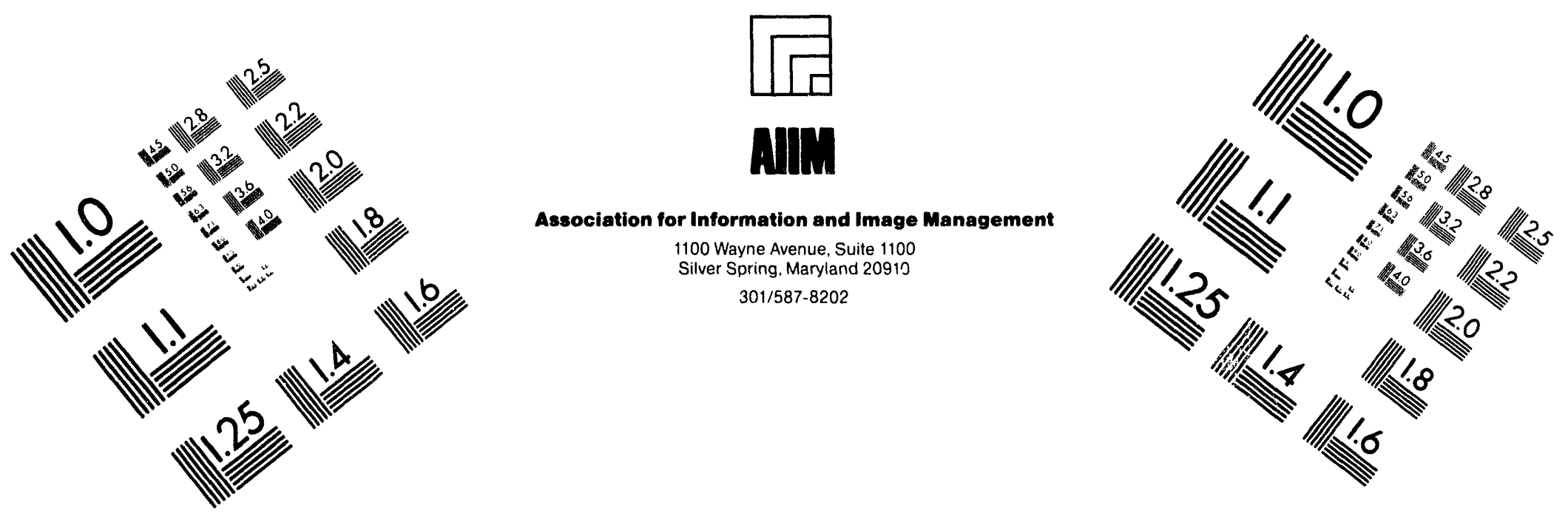

\title{
Centimeter
}

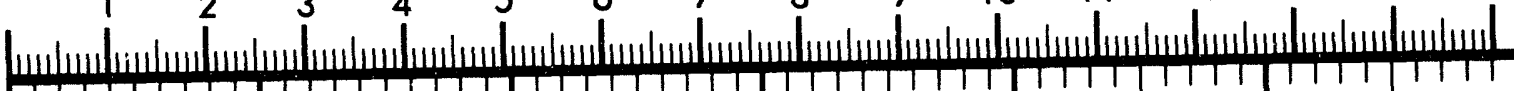

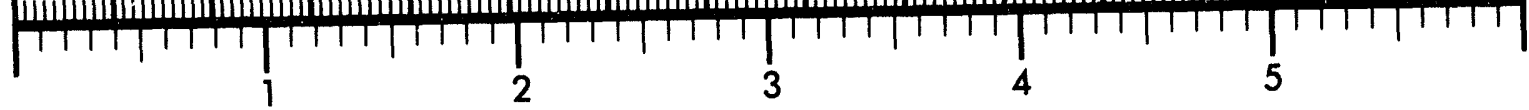
Inches

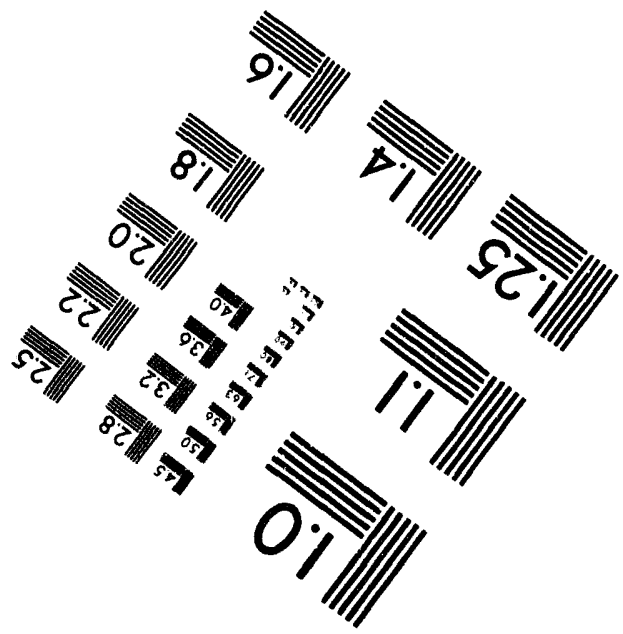

MANUFACTURED TO AIIM STANDARDS

BY APPLIED IMAGE, INC.

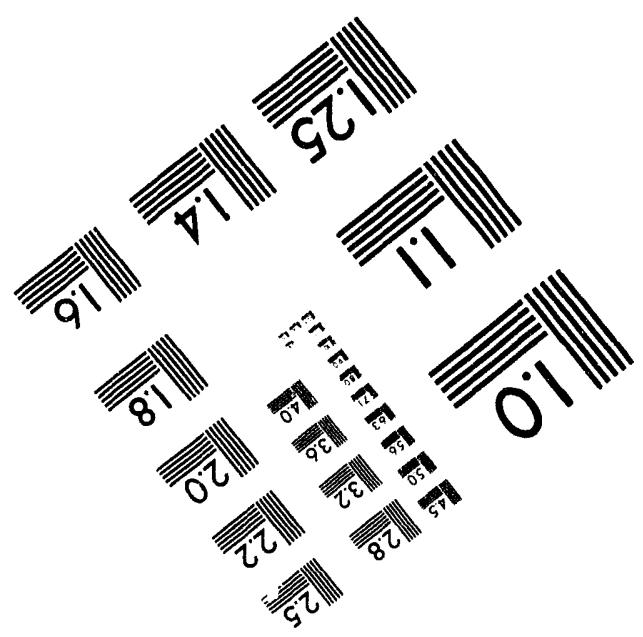



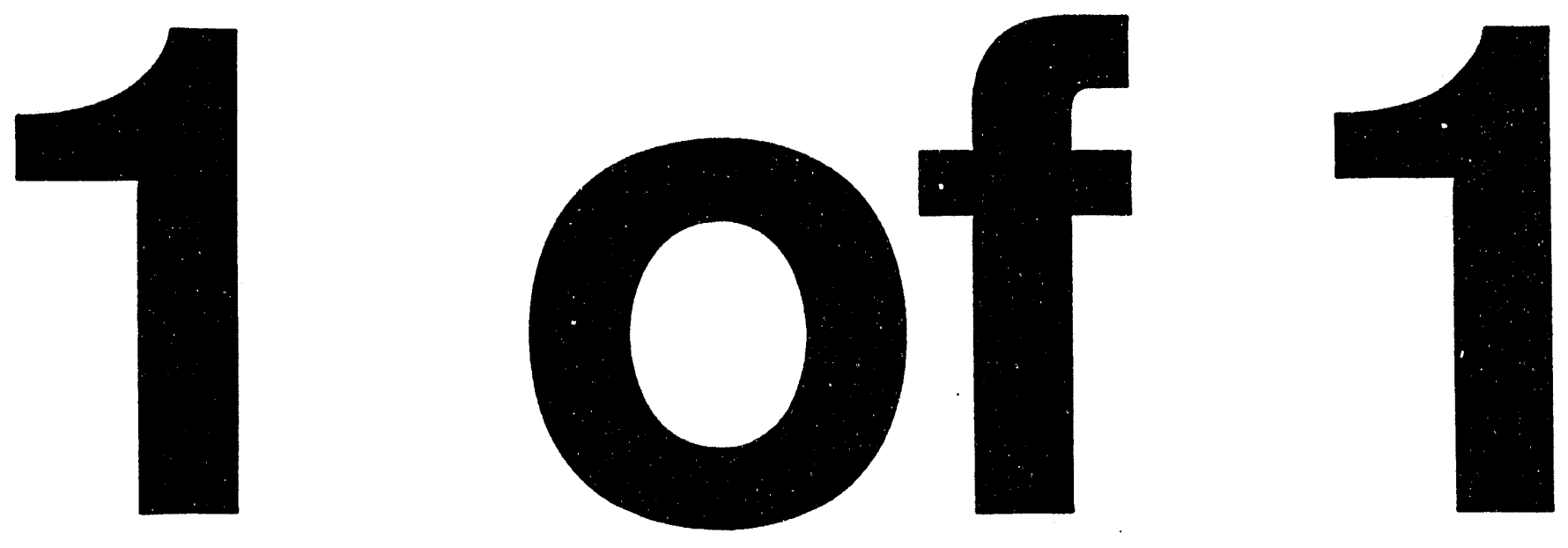


\section{Point Based Hydrodynamics Applied to Shallow Water Equations}

\section{Introduction}

Smoothed Particle Hydrodynamics (SPH) has proven useful for many problems of scientific interest. This report is concerned with a generalization to the SPH method which may be of value for problems such as climate modeling. The main objective of the work is to carry out the new formulation, called Point Interactive Physics (PIP), and to compare results for PIP and standard finite difference techniques on a simple test problem.

The basic SPH approach (Monaghan, 1992), (Benz, 1989), (Cloutman, 1990) describes a system using a set of points. These points are not associated with a grid or mesh, as in most approaches. The individual points are each assigned a mass and move with the material velocity. Thus the original SPH method is Lagrangian. Properties of the system are expressed as sums over the sample points using a weighting function called a kernel function. For most problems, the weighting function extends over a relatively short range, called the smoothing length.

The Point Interacting Physics (PIP) approach also samples information at a set of points, and then uses kernel functions to infer physical properties everywhere. The points are not assigned fixed values of mass and they are allowed to undergo arbitrary physically reasonable motion. In this report, results will be presented for fixed points arranged irregularly over a spherical surface. Thus, this version of PIP will be applied in an Eulerian fashion. The basic reason for the Eulerian treatment is enhanced efficiency arising from the fixed geometric relationships among the points.

After a brief description of the SPH and PIP methods, the PIP formulation will be compared to a normal finite difference approach for a simple shallow water advection test problem. It appears that accurate simulations can be accomplished, but that computational speed is comparable to that observed for other techniques.

Classical Smoothed Particle Hydrodynamics (SPH)

The SPH approach has been described in a number of references (Benz, 1989). The basic equation for density is

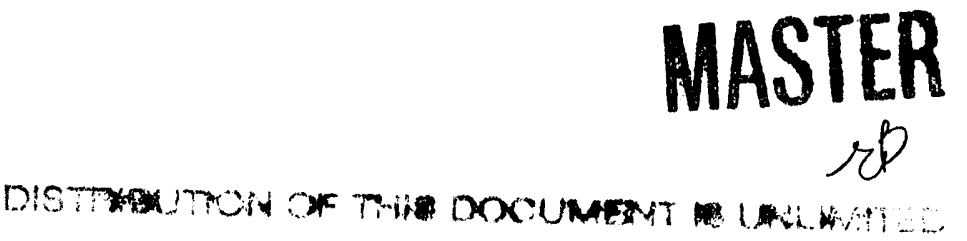




$$
\langle\rho(\vec{r})\rangle=\sum_{j} m_{j} W\left(\vec{r}-\vec{r}_{j}, h\right)
$$

where the angle brackets signify the expectation value for the enclosed quantity. $\vec{r}$ is the spatial position vector, and $m_{j}$ is the mass of the particle at position $\vec{r}_{j} . h$ is the smoothing length, which is a measure of the distance over which a particle's influence is felt. The summation is carried out over all particles in the system, but, because the kernel function $W$ has a finite range, the sum actually extends only over a set of points which are in the neighborhood of $\vec{r}$. The expectation value of any field $A$ can be obtained from the expression

$$
\langle A(\vec{r})\rangle=\sum_{j} m_{j} \frac{A_{j}}{\rho_{j}} W\left(\vec{r}-\vec{r}_{j}, h\right)
$$

where $A_{j}$ is the observed value of the field at point j. For hydrodynamics, the field $A$ can represent velocity or energy.

The mass density update can be carried out explicitly or inferred from the motion of the points. Both methods are used in practice.

A typical equation for velocity update at particle $i$ is

$$
\frac{d \vec{v}_{i}}{d t}=-\sum_{j} m_{j}\left(\frac{P_{i}}{\rho_{i}^{2}}+\frac{P_{j}}{\rho_{j}^{2}}\right) \nabla_{i} W\left(\vec{r}_{i}-\vec{r}_{j}, h\right)
$$

where $t$ is time, $\vec{v}_{i}$ is the velocity of particle $\mathrm{i}$, and $P$ is piessure. The specific form of Equation (3) is chosen to achieve momentum conservation and symmetric momentum exchange between particles. It also conserves angular momentum. When converted to a difference equation, the time differencing is linear.

An equation for the specific internal energy update is

$$
\frac{d \varepsilon_{m i}}{d t}=-\frac{P_{i}}{\rho_{i}^{2}} \sum_{j} m_{j}\left(\vec{v}_{i}-\vec{v}_{j}\right) \cdot \nabla_{i} W\left(\vec{r}_{i}-\vec{r}_{j}, h\right)
$$


where $\varepsilon_{m}$ is specific internal energy.

The most popular kernel function is the following cubic.

$$
W(\vec{r}, h)=\frac{1}{\pi h^{3}} \begin{cases}1-(3 / 4) d^{2}(2-d), & d<1 \\ (1 / 4)(2-d)^{3}, & 1 \leq d<2 \\ 0, & d \geq 2\end{cases}
$$

where $d=\left|\frac{\vec{r}}{h}\right|$.

\section{Point Interacting Physics (PIP)}

Expectation values for density, momentum, and energy are the same as for SFH. See Equation (2).

The update equations have three basic components: transport, divergence / convergence, and source terms. Because the method is not Lagrangian, the density update must be explicitly carried out. The main focus of the present work is on problems without source terms, so they will not be shown here.

The PIP method allows a different approach to the problem of conducting time updates. Pure transport is handled by space-time displacement, divergence by evaluation along the spatial path, and source terms by time and space centered evaluation. The updated mass density is given by

$$
\rho\left(\vec{r}_{i}, t+\Delta t\right)=\rho\left(\vec{r}_{i}-\vec{v}_{i} \Delta t, t\right)+\rho\left(\vec{r}_{i}, t\right) \nabla \cdot \vec{v}_{i} \Delta t
$$

where $\vec{r}_{i}$ in the density terms is evaluated at the times shown. This approach is classed as semi-Lagrangian. Given the density from Eq. (6), the masses are then found, using the same approach as at problem initialization. 


$$
m_{i}=\rho_{i}^{2} / \sum_{j} \rho_{j} W_{i j}
$$

\section{Shallow Water Test}

The PIP approach was tested using a simple formulation based on shallow water theory applied to a rotating sphere. This is a standard method used to calibrate many types of codes used in weather prediction and climate modeling. A suite of test problems has been published by Williamson, et al. (Williamson, 1992). Solutions for spectral transform methods have been published by Jakob, et al. (Jakob, 1993).

A comparison has been made between the PIP code and a "state-of-the-art" finite differences code used for global climate studies at LLNL, called CAMILLE. Results are reported here for test problem 1 of the Williamson suite. A "hill" is centered on the earth's equator, at longitude $+90^{\circ}$ (east), and is advected by a specified velocity field completely around the earth in 12 days. The desired result is that the situation after 12 days should reproduce the initial state.

\section{Code Formulations}

The CAMILLE atmospheric general circulation model (representative of grid point Eulerian techniques) uses finite difference techniques for both second and fourth order solutions to the advection problem on a logically rectangular regular mesh. The code is written in FORTRAN and uses arrays to take advantage of the regularity of the data structures. It was executed with two height levels. The vorticity calculation was skipped. Altogether, five quantities were advected. The basic code logic advances the entire problem using a fixed time step loop within which loops over the all spatial zones are executed.

The PIP model (described above) uses a generalization of SPH techniques on a set of points which have roughly equidistant spacing on the surface of the sphere. The code is written in C and uses pointers and lists to manage the irregular sets of points which sample a region. Three quantities were advected. The basic code logic schedules each point for independent advance. The time step was held fixed for problems reported on here. 
Both codes actually solved the full set of Navier-Stokes equations. The updated velocity fields were overwritten by specified values for test problem 1 . Energy values were computed but ignored.

\section{Code Performance}

Timing results for PIP and CAMILLE obtained on the BBN TC2000 computer at LLNL. All runs were on a single processor, although both codes were capable of running on multiple processors. Code sections were timed using standard UNIX timing tools.

Equatorial Shallow Water Depth

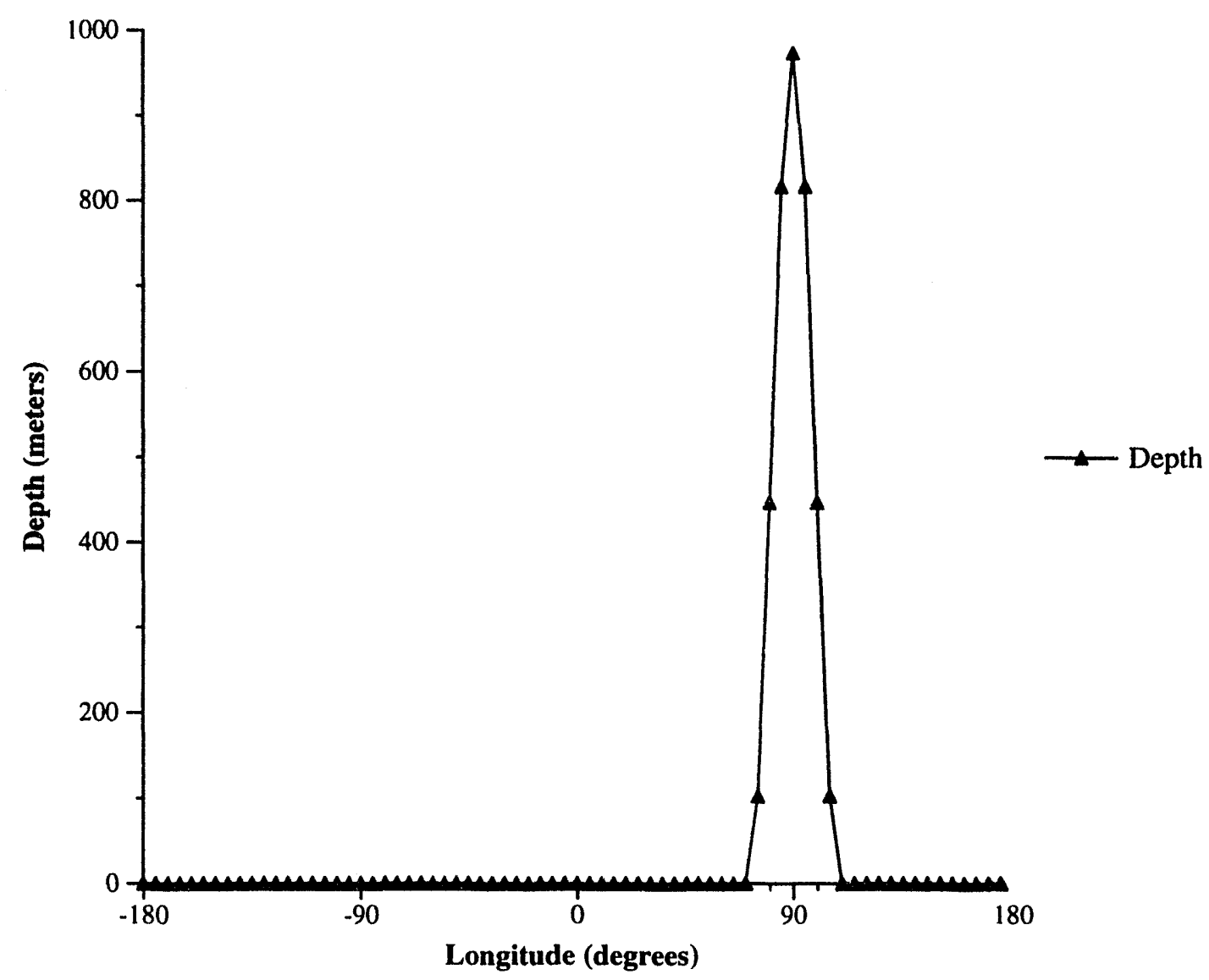

Figure 1. Original shallow water depth profile along the equator for advection test problem.

The CAMILLE code used a grid spacing of 5 degrees in longitude by 4 degrees in latitude, yielding $72 \times 45=3240$ points. The PIP code used an irregular set of 2084 points with average spacing corresponding to 5 degrees of latitude. 


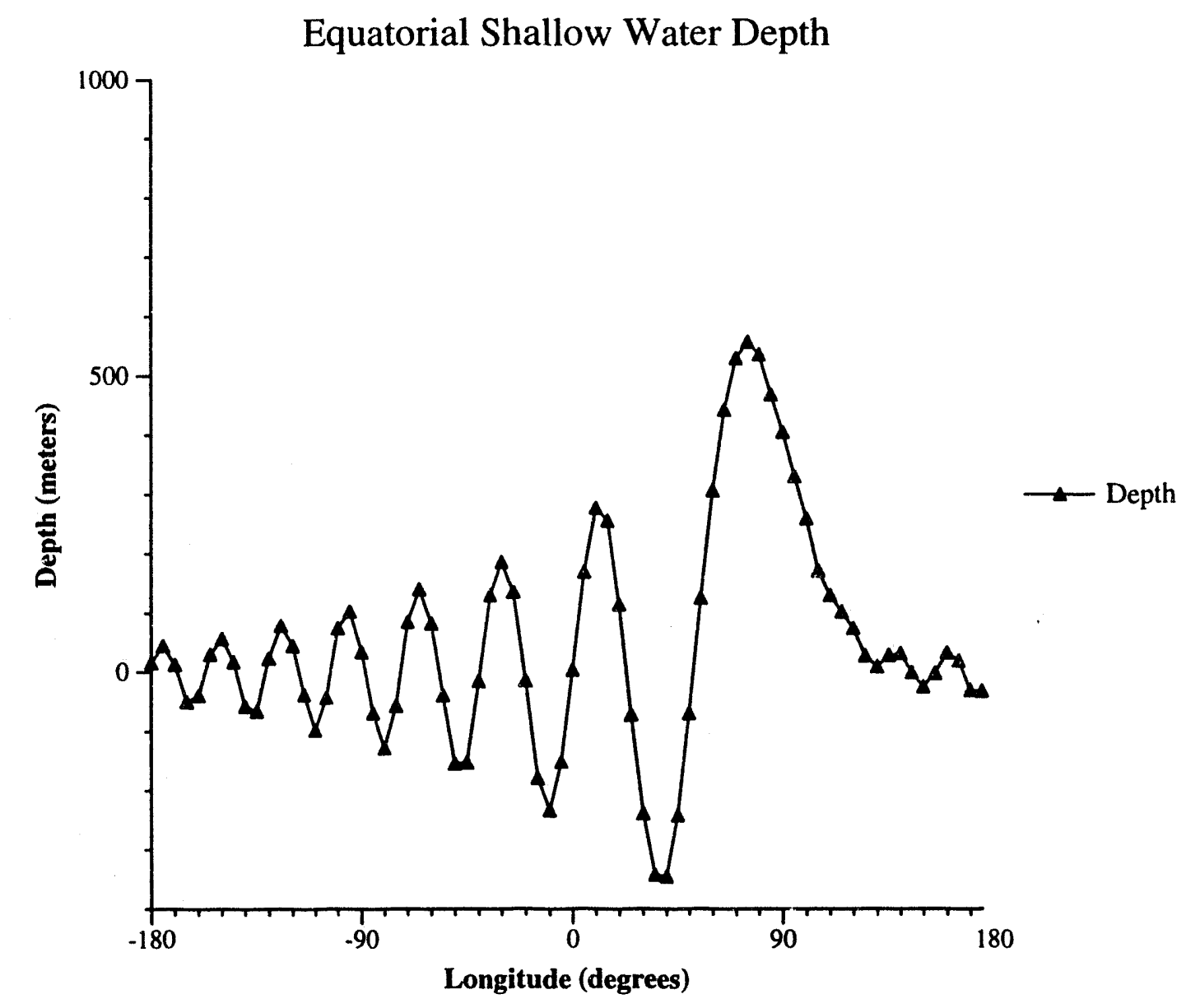

Figure 2. Shallow water depth profile along the equator for advection test problem after 12 days using second order advection in the CAMILLE code. A perfect advection implementation would yield results identical to the starting configuration shown in Figure 1.

The overall accuracy of the PIP code for the advection problem was better than that for the standard finite difference code. However, for climate simulations, other processes will set spatial accuracy requirements, so timing comparisons were carried out for comparable spatial distributions of points, without attempting to determine how few PIP points would be needed to produce acceptable advection. On the other hand, the time steps for the codes were very different. The CAMILLE code was run with hydrodynamics time step set to 450 seconds. The PIP code was run with time step 14400 seconds (one-sixth of a day, corresponding to a rigid body translation of five latitudinal degrees with the given 
velocity field.) Attempts to run CAMILLE with large timesteps led to instability in the fourth order advection.

Equatorial Shallow Water Depth

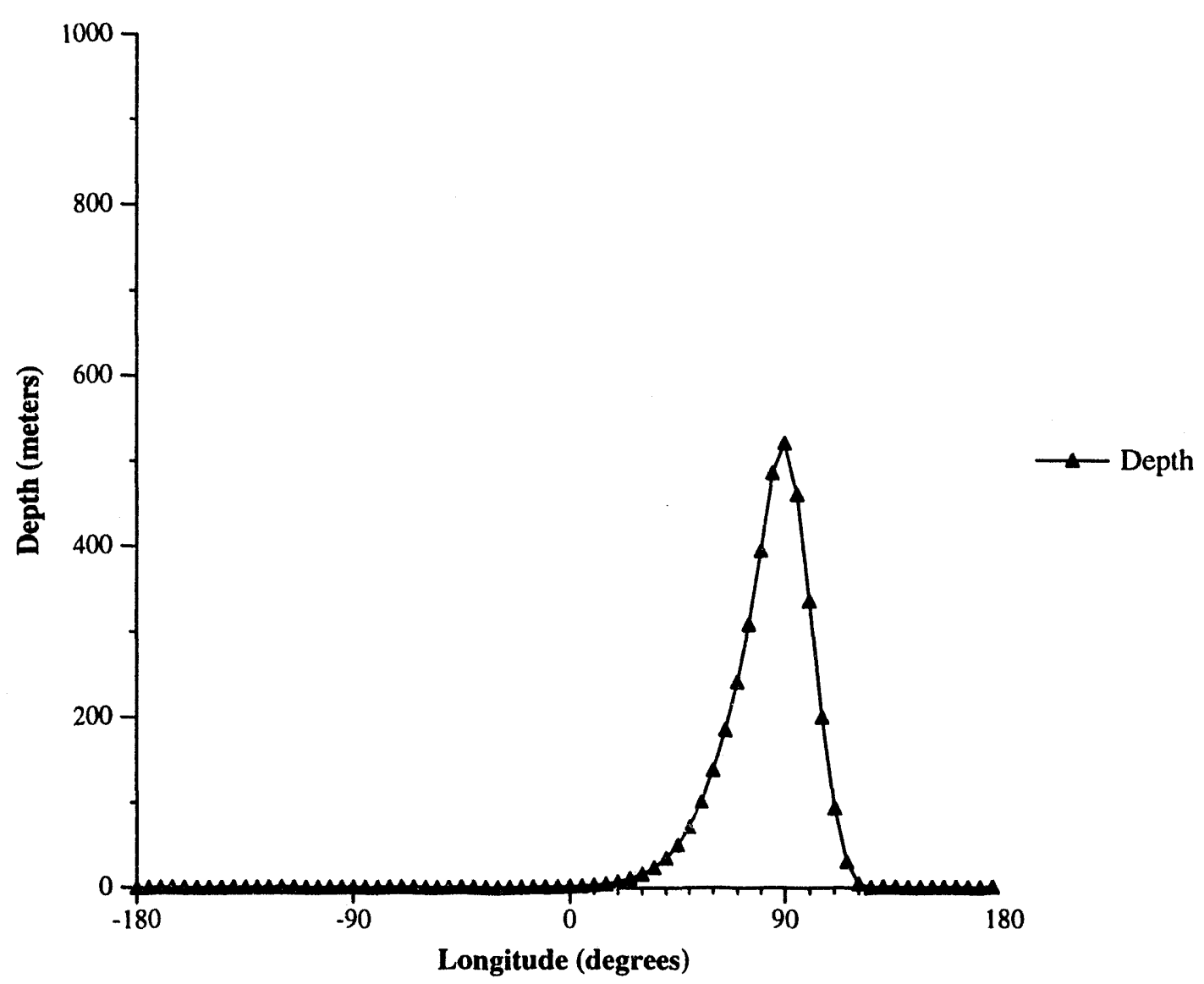

Figure 3. Shallow water depth profile along the equator for advection test problem after 12 days using fourth order advection in the CAMILLE code. A perfect advection implementation would yield results identical to the starting configuration shown in Figure 1.

The question of spatial accuracy is somewhat muddled, since there is no direct comparison between the two codes. There are at least two aspects to the matter. The first is the degree to which the kernel function yields an acceptable representation of the physical distributions in the problem. The second is the extent of the points which contribute to an update. In the PIP formulation, contributions are possible from points corresponding to neighbors of neighbors, leading to the conclusion that fourth order spatial accuracy is possible, without proving that it is actually accomplished. In any 
event, the PIP results show very good agreement with expectations for the shallow water advection test problem.

Equatorial Shallow Water Depth

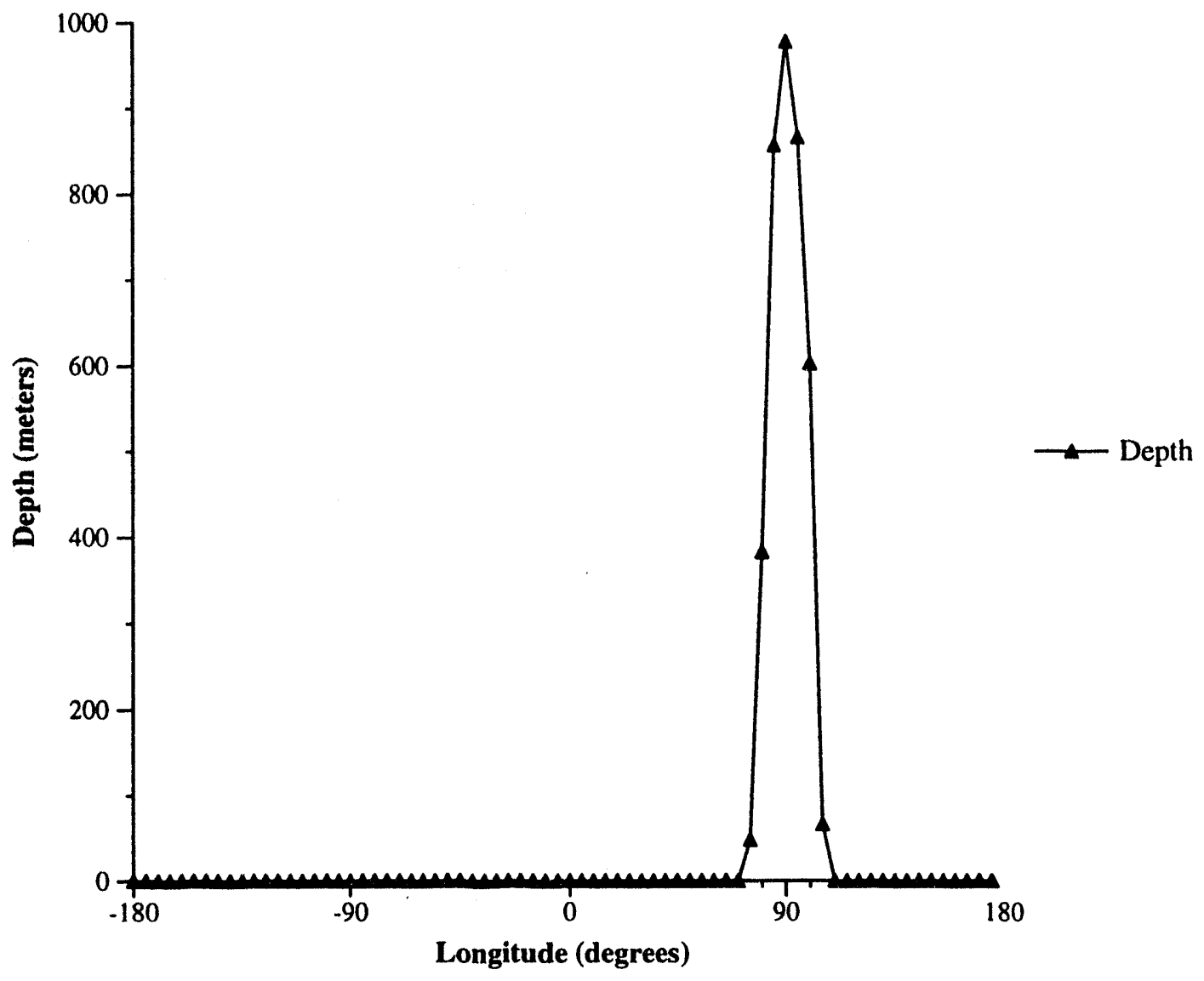

Figure 4. Shallow water depth profile along the equator for advection test problem after 12 days using semi-Lagrange advection in the PIP code.

Figures 1 through 4 show depth profiles for the shallow water equatorial advection test problem. It is clear that neither CAMILLE scheme is particularly suitable for pure advection, but the schemes have been optimized for vorticity and enstrophy treatment. The PIP results seem quite good for a finite difference code.

For the results given above, the CAMILLE and PIP codes consume approximately the same amount of time, namely 300 and 400 seconds, respectively, for the twelve day test period on a BBN TC 2000 computer at LLNL. This computer does not support vectorization. Both codes accomplished the same calculation, but they were constructed in very different ways. The CAMILLE code was 
written in "classic" FORTRAN, using arrays as the basic data structures. The PIP code was written in C, using lists to manage the irregular data sets present in SPH. Because of these and other differences, it is difficult to make a definitive comparison between the two. However, since in other contexts SPH codes have been shown to have significant speed advantages over finite difference codes, it is meaningful to note that, at similar spatial resolutions for test problems in global climate modeling, there appears to be no such advantage.

A major reason behind the performance results for the PIP code was the large number of distance calculations which had to be performed on a spherical surface. These calculations involved many trigonometric functions, which consumed a large fraction of the measured run times. This penalty could be overcome by using standard SPH practice of precomputing distances, storing into a table, and looking up results.

\section{Conclusions}

The SPH approach, as implemented in the PIP code, yields a very accurate, simple, and robust computational system which can be applied to global climate modeling problems. However, the execution time for the PIP code is approximately the same as for other, more standard, approaches. Therefore, there appears to be no compelling benefit resulting from the use of SPH schemes, unless considerations of increased accuracy or spatial resolution outweigh the advantages of present day grid point methods. 


\section{References}

Benz, W. (1989). Smooth Particle Hydrodynamics: A Review (Center for Astrophysics Preprint No. 2884). Harvard-Smithsonian Center for Astrophysics.

Cloutman, L. D. (1990). Basics of Smoothed Particle Hydrodynamics No. UCRL-ID-103698). Lawrence Livermore National Laboratory.

Jakob, R., J. J. Hack, and D. L. Williamson (1993). Solutions to the Shallow Water Test Set Using the Spectral Transform Method (Technical Note No. NCAR/TN-388+STR). NCAR.

Monaghan, J. J. (1992). Smoothed Particle Hydrodynamics. Annual Rev. Astron. Astrophys., 30, 543-574.

Williamson, D. L., J. B. Drake, J. J. Hack, R. Jakob, and P. N. Swarztrauber (1992). A Standard Test Set for Numerical Approximations to the Shallow Water Equations in Spherical Geometry. Journal of Computational Physics, 102(1), 211-224. 

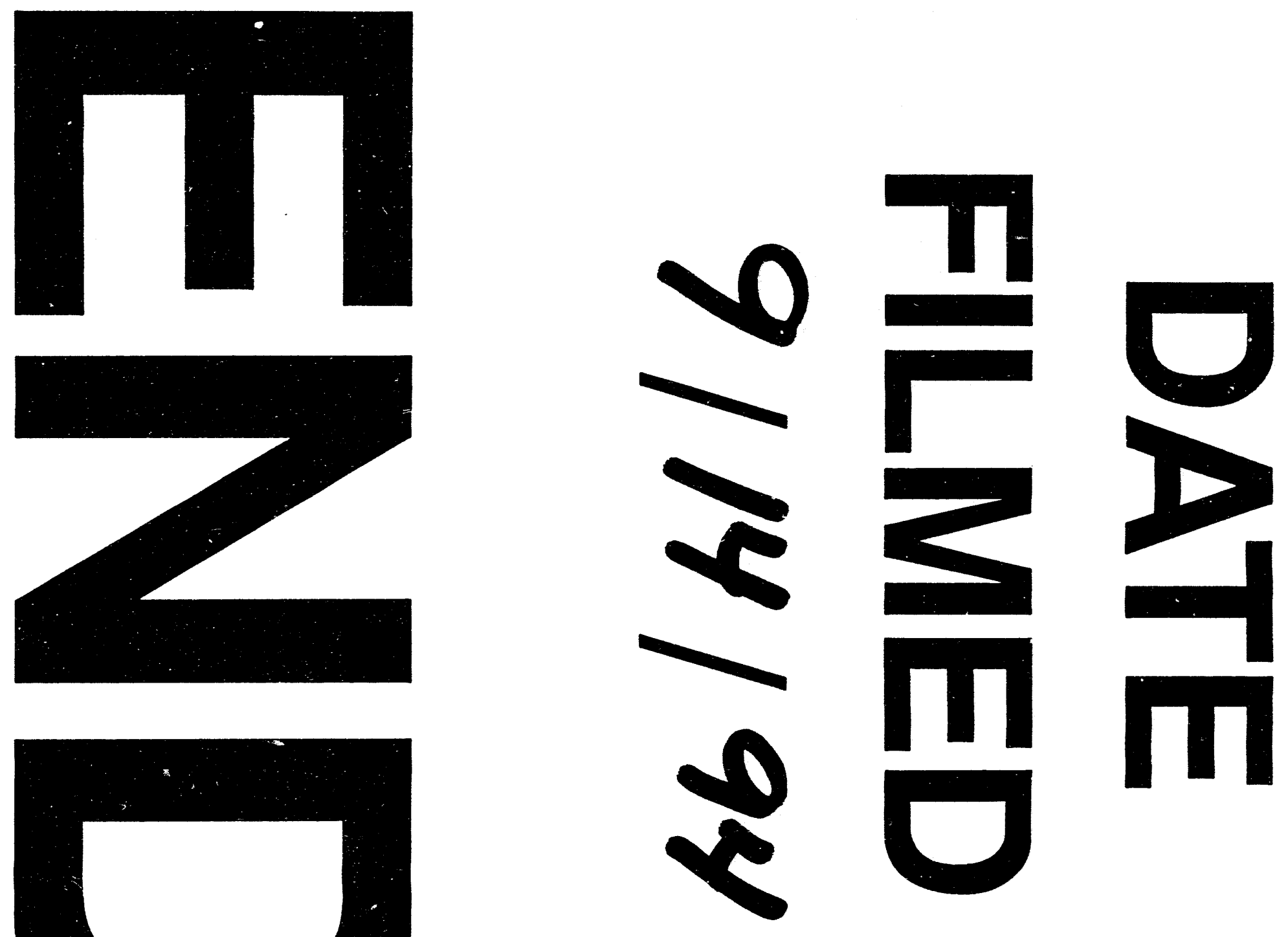

서

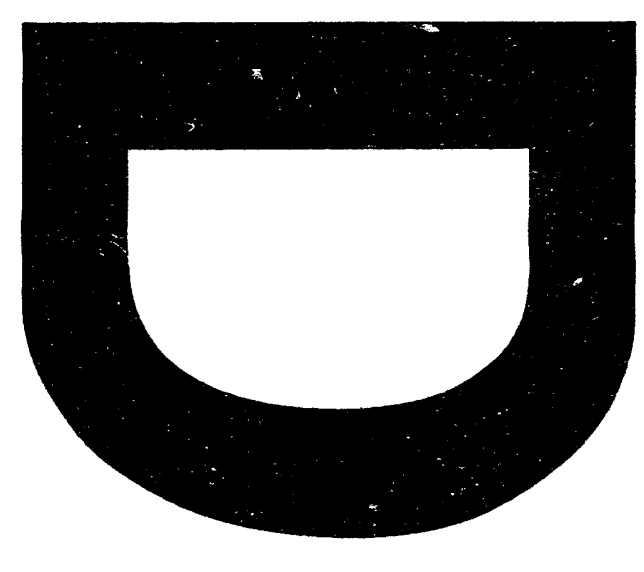

